\title{
ON $q$-SERIES IDENTITIES FOR FALSE THETA SERIES
}

\author{
CHRIS JENNINGS-SHAFFER AND ANTUN MILAS
}

\begin{abstract}
We prove several infinite families of $q$-series identities for false theta functions and related series. These identities are motivated by considerations of characters of modules of vertex operator superalgebras and of quantum dilogarithms. We also obtain closely related modular identities of the Göllnitz-Gordon-Andrews type. As a byproduct of our identities, we establish several identities for the Rogers dilogarithm function coming from multi $q$ hypergeometric series with "double poles".
\end{abstract}

\section{INTRODUCTION}

False theta functions are similar to theta functions except for an alteration of the signs in some of the series terms, which prevents them from being modular forms. They appear in a variety of contexts including modular and quantum modular forms [16, 23, 35], meromorphic Jacobi forms [12, quantum knot invariants [10, 24, 27, 30, 33] (e.g. tails of colored Jones polynomials), Witten-Reshetikhin-Turaev invariants of Seifert 3-manifolds [13, 28, 29], and many more. More recently, they have appeared in the context of "homological blocks" of $3 \mathrm{~d}$-theories $T[M]$ with the gauge group $S U(2)$, where $M$ is a plumbed 3-manifold [26] (see also [15, 18]). There are also higher-rank generalizations of false theta functions introduced and studied by K. Bringmann, T. Creutzig, and the second author [17, 20] (see also [14]).

The literature on $q$-series identities connecting partial and false theta functions with $q$ hypergeometric series is extensive (see [43] and references therein). One of the most elegant identities in this context was given by Ramanujan [11, p. 18]

$$
\sum_{n \in \mathbb{Z}} \operatorname{sgn}(n) q^{2 n^{2}+n}=(q)_{\infty} \sum_{n \geq 0} \frac{q^{n^{2}+n}}{(q)_{n}^{2}},
$$

where $\operatorname{sgn}(n):=1$ for $n \geq 0$ and -1 otherwise (as usual, $(a)_{n}:=\prod_{i=1}^{n}\left(1-a q^{i-1}\right)$ ). The $q$-series on the left-hand side is an example of Rogers' false theta function.

Ramanujan's identity can be generalized to all positive integral characteristics.

Theorem 1.1 ([17, 27, 46]). For $k \in \mathbb{N}$, we have

$$
\frac{q^{-\frac{k^{2}}{4(k+1)}}}{(q)_{\infty}} \sum_{n \in \mathbb{Z}} \operatorname{sgn}(n) q^{(k+1)\left(n+\frac{k}{2(k+1)}\right)^{2}}=\sum_{n_{1}, n_{2}, \ldots, n_{k} \geq 0} \frac{q^{N_{1}^{2}+N_{2}^{2}+\cdots+N_{k}^{2}+N_{1}+N_{2}+\cdots+N_{k}}}{(q)_{n_{k}}^{2}(q)_{n_{1}}(q)_{n_{2}} \cdots(q)_{n_{k-1}}},
$$

where $N_{i}=\sum_{j \geq i} n_{j}$.

This theorem can be approached in several different ways; it follows from the analytic form of the Andrews-Gordon identities combined with standard $q$-summation techniques [16], or using relations for the tail of colored Jones polynomials of $(2,2 k)$ torus knots [27], or using Bailey's Lemma [43, 46]. From the vertex algebra perspective, this particular form is interesting because the left-hand side in (1.2) is the character of the $(1, k+1)$-singlet vertex algebra [17]; this explains a somewhat unusual shift in the exponent and the Euler factor 
appearing in the denominator (cf. (1.1)). For additional aspects of partial theta and theta functions, we refer the reader to the recent review article by O. Warnaar [45] and references therein; see also [22, 44] for related identities in the context of conformal field theory.

More recently, in [1, 2, 39], a different type of false theta function was (re)discovered in connection with the $N=1$ super-singlet vertex superalgebras. Characters of irreducible modules of the super-singlet are similar to the expression in (1.2), but they contain a "fermionic" product $\left(-q^{\frac{1}{2}}\right)_{\infty}$ and their characteristic is half-integral $\left(k \in \mathbb{N}, \epsilon \in\left\{0, \frac{1}{2}\right\}\right)$ :

$$
\frac{\left(-q^{\frac{1}{2}+\epsilon}\right)_{\infty}}{(q)_{\infty}} \sum_{n \in \mathbb{Z}} \operatorname{sgn}(n) q^{\left(k+\frac{1}{2}\right)(n+a)^{2}}
$$

for some $a \in \mathbb{Q}$. Regularized modular properties of these expressions have been thoroughly investigated in [3, 39].

Characters of modules for the $N=1$ super-singlet algebra combine into characters of modules for certain "logarithmic" vertex algebras called $N=1$ super-triplets [2]. Their irreducible characters take the following form (see [1, 2])

$$
\frac{\left(-q^{\frac{1}{2}+\epsilon}\right)_{\infty}}{(q)_{\infty}} \sum_{n \in \mathbb{Z}}(2 n+1) q^{\left(k+\frac{1}{2}\right)(n+a)^{2}} .
$$

This series, up to a multiplicative $q$-power, is known to be modular. More precisely, the theta-like series in (1.4) is a sum of modular forms of weight $\frac{1}{2}$ and $\frac{3}{2}$.

Although the origin of false theta functions is not completely understood, their primary source is usually attributed to meromorphic Jacobi forms [12, Chapter 11]. The simplest instance of this arises in the following well-known Fourier expansion [6, 41] $\left(|q|<|\zeta|^{2}<1\right)$ :

$$
\frac{1}{\left(\zeta q^{\frac{1}{2}}, \zeta^{-1} q^{\frac{1}{2}}\right)_{\infty}}=\frac{1}{(q)_{\infty}^{2}} \sum_{n_{1}} \sum_{n_{2} \geq\left|n_{1}\right|}(-1)^{n_{1}+n_{2}} q^{\frac{n_{2}\left(n_{2}+1\right)}{2}-\frac{n_{1}^{2}}{2}} \zeta^{n_{1}}=\sum_{\substack{n_{1} \in \mathbb{Z} \\ n_{2} \geq 0}} \frac{q^{\frac{\left|n_{1}\right|}{2}+n_{2}}}{(q)_{n_{2}}(q)_{\left|n_{1}\right|+n_{2}}} \zeta^{n_{1}},
$$

where $\left(a_{1}, \ldots, a_{k}\right)_{m}:=\left(a_{1}\right)_{m} \cdots\left(a_{k}\right)_{m}$. As is plainly seen, extracting the constant term with respect to $\zeta$ yields another elegant expression for the Rogers false theta function (with $k=1$ ),

$$
\frac{1}{(q)_{\infty}^{2}} \sum_{n \in \mathbb{Z}} \operatorname{sgn}(n) q^{2 n^{2}+n}=\sum_{n \geq 0} \frac{q^{n}}{(q)_{n}^{2}}
$$

Compared to formula (1.1), this identity comes with an extra Euler factor and has no quadratic term in the exponent of the $q$-hypergeometric term.

The main purpose of this paper is to motivate and prove:

(I) An analog of (1.2) for certain $q$-series as in (1.3). In particular, this would give a combinatorial form for irreducibile characters of $N=1$ super-singlet modules.

(II) A Göllnitz-Andrews-Gordon-type expression for the $q$-series in (1.3). Related identities in a non-super context were studied in [44].

(III) A family of $q$-series identities for false theta series coming from Fourier expansions of multi-variable Jacobi forms, in parallel to the $k=1$ case seen in (1.6).

Let us briefly outline our solutions to these problems and the contents of the paper.

In Section 2, we gather several facts on Bailey pairs and Bailey chains used in the paper. These powerful methods are applied in Section 3 to prove identities for "shifted" false theta 
functions. In Proposition 3.1, we present identities for $\epsilon=0$ whereas Proposition 3.2 pertains to $\epsilon=\frac{1}{2}$. As a special case, we obtain $q$-hypergeometric expressions for (1.3).

In Section 4, we first use Lemma 4.1, to write (1.4) as an infinite sum of shifted false theta series. Then we combine this with identities from Section 3, and obtain a single multihypergeometric series with a parity condition. Our main results here are Theorems 4.2, 4.4, and 4.5, with Theorem 4.2 corresponding to $\epsilon=0$ and Theorems 4.4 and 4.5 corresponding to $\epsilon=\frac{1}{2}$.

In Section 5, we switch our focus to $q$-series identities generalizing (1.6); see Theorem 5.1. We first express the relevant $q$-hypergometric series in a form convenient for use of Bailey chains from Section 2 (see Lemma 5.3). Interestingly, this methods allows us to prove more than we hoped for; we also obtain a family of modular identities in Theorem 5.2 that intertwine with the "false" identities in Theorem 5.1. We should point out that Theorem 5.2 was recently conjectured in [19] in connection to Argyres-Douglas theories in physics.

In Section 6, using well-established methods [34, 42, 48, we prove several identities for the Rogers dilogarithm function (see Propositions 6.2, 6.3, and 6.4). We finish in Section 7 with a few pointers for future research.

Acknowledgments. We thank C. Nazaroglu for pointing out [19]. We also thank O. Warnaar, J. Lovejoy, R. Osburn and K. Bringmann for informative discussions and suggestions. Lastly, we thank the anonymous referees for their various corrections and improvements to an earlier version of this manuscript.

\section{Bailey pairs, the Bailey Chain, And the Bailey Lattice}

We recall a pair of sequences $\left(\alpha_{n}, \beta_{n}\right)$ is called a Bailey pair relative to $(a, q)$ if

$$
\beta_{n}=\sum_{j=0}^{n} \frac{\alpha_{j}}{(q)_{n-j}(a q)_{n+j}} .
$$

Bailey's lemma is an identity relating rather general $q$-series involving Bailey pairs. It was introduced by W. N. Bailey [9] to give a unified framework to reprove the Rogers-Ramanujan identities as well as several related identities of Rogers. The "Bailey pair machinery" refers to a combination of Bailey pairs, Bailey's lemma, and various generalizations that were largely made famous by G. Andrews. From the Bailey pair machinery, our proofs require the so-called Bailey chain and Bailey lattice, rather than just Bailey's lemma.

The full version of the $k$-fold iteration of Bailey's lemma can be found as Theorem 3.4 in [7]. We let the parameters $N \rightarrow \infty, b_{1}=b_{2}=\cdots=b_{k} \rightarrow \infty, c_{1}=c_{2}=\cdots=c_{k}=q$, and specialize to Bailey pairs with $a=q$. By doing so we have that

$$
\begin{aligned}
& \sum_{n_{k} \geq n_{k-1} \geq \cdots \geq n_{1} \geq 0} \frac{(q)_{n_{1}}(-1)^{n_{1}+n_{2}+\cdots+n_{k}} q^{\frac{n_{1}\left(n_{1}+1\right)}{2}+\frac{n_{2}\left(n_{2}+1\right)}{2}+\cdots+\frac{n_{k}\left(n_{k}+1\right)}{2}} \beta_{n_{1}}}{(q)_{n_{k}-n_{k-1}}(q)_{n_{k-1}-n_{k-2}} \cdots(q)_{n_{2}-n_{1}}} \\
&=(1-q) \sum_{n \geq 0}(-1)^{n k} q^{\frac{k n(n+1)}{2}} \alpha_{n},
\end{aligned}
$$

for Bailey pairs $\left(\alpha_{n}, \beta_{n}\right)$ relative to $(q, q)$. 
The statement for the Bailey lattice is given in [4] as Theorem 3.1. We let $n \rightarrow \infty, \rho_{1}=\rho$, $\rho_{j} \rightarrow \infty$ for $2 \leq j \leq k, \sigma_{j} \rightarrow \infty$ for $1 \leq j \leq k$. The identity then becomes

$$
\begin{aligned}
& \sum_{m_{1} \geq m_{2} \geq \cdots \geq m_{k} \geq 0} \frac{(\rho)_{m_{1}}(-1)^{m_{1}} \rho^{-m_{1}} a^{m_{1}+m_{2}+\cdots+m_{k}} q^{\frac{m_{1}\left(m_{1}-1\right)}{2}+m_{2}^{2}+m_{3}^{2}+\cdots+m_{k}^{2}-m_{2}-m_{3}-\cdots-m_{i}} \beta_{m_{k}}}{(q)_{m_{1}-m_{2}}(q)_{m_{2}-m_{3}} \cdots(q)_{m_{k-1}-m_{k}}} \\
= & \frac{\left(a \rho^{-1}\right)_{\infty}}{(a q)_{\infty}} \sum_{n \geq 0} \frac{(\rho)_{n}(-1)^{n} \rho^{-n} a^{k n} q^{\left(k-\frac{1}{2}\right) n^{2}+\left(\frac{1}{2}-i\right) n} \alpha_{n}}{\left(a \rho^{-1}\right)_{n}\left(1-a q^{2 n}\right)} \\
& -\frac{\left(a \rho^{-1}\right)_{\infty}}{(a q)_{\infty}} \sum_{n \geq 1} \frac{(\rho)_{n}(-1)^{n} \rho^{-n} a^{k n+i-k+1} q^{\left(k-\frac{1}{2}\right) n^{2}+\left(\frac{5}{2}+i-2 k\right) n+k-i-2} \alpha_{n-1}}{\left(a \rho^{-1}\right)_{n}\left(1-a q^{2 n-2}\right)},
\end{aligned}
$$

where $0 \leq i \leq k$ and $\left(\alpha_{n}, \beta_{n}\right)$ is Bailey pair relative to $(a, q)$. We further modify this identity by reindexing with $m_{j}=N_{j}=n_{j}+n_{j+1}+\cdots n_{k}$, which gives

$$
\begin{aligned}
& \sum_{n_{1}, n_{2}, \ldots, n_{k} \geq 0} \frac{(\rho)_{N_{1}}(-1)^{N_{1}} \rho^{-N_{1}} a^{N_{1}+N_{2}+\cdots+N_{k}} q^{\frac{N_{1}\left(N_{1}-1\right)}{2}+N_{2}^{2}+N_{3}^{2}+\cdots+N_{k}^{2}-N_{2}-N_{3}-\cdots-N_{i}} \beta_{n_{k}}}{(q)_{n_{1}}(q)_{n_{2}} \cdots(q)_{n_{k-1}}} \\
= & \frac{\left(a \rho^{-1}\right)_{\infty}}{(a q)_{\infty}} \sum_{n \geq 0} \frac{(\rho)_{n}(-1)^{n} \rho^{-n} a^{k n} q^{\left(k-\frac{1}{2}\right) n^{2}+\left(\frac{1}{2}-i\right) n} \alpha_{n}}{\left(a \rho^{-1}\right)_{n}\left(1-a q^{2 n}\right)} \\
& -\frac{\left(a \rho^{-1}\right)_{\infty}}{(a q)_{\infty}} \sum_{n \geq 1} \frac{(\rho)_{n}(-1)^{n} \rho^{-n} a^{k n+i-k+1} q^{\left(k-\frac{1}{2}\right) n^{2}+\left(\frac{5}{2}+i-2 k\right) n+k-i-2} \alpha_{n-1}}{\left(a \rho^{-1}\right)_{n}\left(1-a q^{2 n-2}\right)} .
\end{aligned}
$$

We note that while it may appear, at first glance, that the right-hand side of (2.2) should follow a different pattern when $i=0$, one can verify with term by term rearrangements that the above is correct.

We require two Bailey pairs. One is the Bailey pair $B(3)$ of Slater [40], which is relative to $(q, q)$ and defined by

$$
\alpha_{n}^{B 3}:=\frac{(-1)^{n} q^{\frac{n(3 n+1)}{2}}\left(1-q^{2 n+1}\right)}{(1-q)}, \quad \quad \beta_{n}^{B 3}:=\frac{1}{(q)_{n}} .
$$

The other Bailey pair we require, which is relative to $(a, q)$, is given by

$$
\alpha_{n}^{\prime}:=\frac{a^{n} q^{n(n-1)}\left(1-a q^{2 n}\right)}{(1-a)}, \quad \beta_{n}^{\prime}:=\frac{1}{(q, a)_{n}} .
$$

That $\left(\alpha_{n}^{\prime}, \beta_{n}^{\prime}\right)$ forms a Bailey pair can be verified in a variety of ways. One method is to take the defining relation of a Bailey pair and simplify the sum on $\alpha_{j}$ with a summation formula for a very well poised ${ }_{6} \phi_{5}$ [25, (II.21)].

\section{False Theta Function Identities}

We start with a family of $q$-series identity for "shifted" false theta functions. These are similar to Rogers' false theta functions, but some of the terms are missing. The first identity is related to the $\epsilon=0$ case discussed in the introduction. 
Proposition 3.1. Suppose $i, k, \ell \in \mathbb{Z}, k \geq 1$, and $0 \leq i \leq k$. Then

$$
\begin{aligned}
& \frac{\left(-q^{\frac{1}{2}}\right)_{\infty}}{(q)_{\infty}}\left(\sum_{n \geq|\ell|}-\sum_{n \leq-|\ell|-1}\right) q^{\left(k+\frac{1}{2}\right)\left(n+\frac{i}{2 k+1}\right)^{2}-\frac{(2 k \ell+i+\ell)^{2}}{4 k+2}} \\
& =\sum_{n_{1}, n_{2}, \ldots, n_{k} \geq 0} \frac{q^{\frac{N_{1}\left(N_{1}+1\right)}{2}+N_{2}^{2}+N_{3}^{2}+\cdots+N_{k}^{2}+\left(\ell+\frac{1}{2}\right) N_{1}+(2 \ell+1)\left(N_{2}+N_{3}+\cdots+N_{k}\right)-N_{1}-N_{2}-\cdots-N_{k-i}\left(-q^{\frac{1}{2}}\right)_{N_{1}+\ell}}}{(q)_{n_{1}}(q)_{n_{2}} \cdots(q)_{n_{k}}(q)_{n_{k}+2 \ell}} .
\end{aligned}
$$

Proof: First we verify that the identity for negative $\ell$ follows from the identity for positive $\ell$. For this, assume the identity holds for positive values of $\ell$. When $\ell$ is negative, $(q)_{n_{k}+2 \ell}^{-1}=0$ for $n_{k}<-2 \ell$, and so

$$
\begin{aligned}
& \sum_{n_{1}, n_{2}, \ldots, n_{k} \geq 0} \frac{q^{\frac{N_{1}\left(N_{1}+1\right)}{2}+N_{2}^{2}+N_{3}^{2}+\cdots+N_{k}^{2}+\left(\ell+\frac{1}{2}\right) N_{1}+(2 \ell+1)\left(N_{2}+N_{3}+\cdots+N_{k}\right)-N_{1}-N_{2}-\cdots-N_{k-i}\left(-q^{\frac{1}{2}}\right)_{N_{1}+\ell}}}{(q)_{n_{1}}(q)_{n_{2}} \cdots(q)_{n_{k}}(q)_{n_{k}+2 \ell}} \\
= & \sum_{n_{1}, n_{2}, \ldots, n_{k} \geq 0} \frac{q^{\frac{\left(N_{1}-2 \ell\right)\left(N_{1}+1-2 \ell\right)}{2}+\left(N_{2}-2 \ell\right)^{2}+\left(N_{3}-2 \ell\right)^{2}+\cdots+\left(N_{k}-2 \ell\right)^{2}\left(-q^{\frac{1}{2}}\right)_{N_{1}-\ell}}}{(q)_{n_{1}}(q)_{n_{2}} \cdots(q)_{n_{k}}(q)_{n_{k}-2 \ell}} \\
= & \times q^{\left(\ell+\frac{1}{2}\right)\left(N_{1}-2 \ell\right)+(2 \ell+1)\left(N_{2}+N_{3}+\cdots+N_{k}-(k-1) 2 \ell\right)-N_{1}-N_{2}-\cdots-N_{k-i}+(k-i) 2 \ell} \\
= & \frac{q^{\frac{N_{1}\left(N_{1}+1\right)}{2}+N_{2}^{2}+N_{3}^{2}+\cdots+N_{k}^{2}+\left(-\ell+\frac{1}{2}\right) N_{1}+(-2 \ell+1)\left(N_{2}+N_{3}+\cdots+N_{k}\right)-N_{1}-N_{2}-\cdots-N_{k-i}-2 i \ell}}{(q)_{n_{1}}(q)_{n_{2}} \cdots(q)_{n_{k}}(q)_{n_{k}-2 \ell}} \\
= & \times(q)_{\infty} q^{\left.\frac{1}{2}\right)_{N_{1}-\ell}}\left(\sum_{n \geq-\ell}-\sum_{n \leq \ell-1}\right) q^{\left(k+\frac{1}{2}\right)\left(n+\frac{i}{2 k+1}\right)^{2}-\frac{(-2 k \ell+i-\ell)^{2}}{4 k+2}-2 i \ell} \\
= & \frac{\left(-q^{\frac{1}{2}}\right)_{\infty}}{(q)_{\infty}}\left(\sum_{n \geq|\ell|}-\sum_{n \leq-|\ell|-1}\right) q^{\left(k+\frac{1}{2}\right)\left(n+\frac{i}{2 k+1}\right)^{2}-\frac{(2 k \ell+i+\ell)^{2}}{4 k+2}} .
\end{aligned}
$$

As such, we need only consider the case where $\ell \geq 0$.

We prove this identity after the change of variable $i \mapsto k-i$. We apply (2.2) with $\rho \mapsto-q^{\ell+\frac{1}{2}}$ to the Bailey pair in (2.4) with $a=q^{2 \ell+1}$. Doing so yields

$$
\begin{aligned}
& \sum_{n_{1}, n_{2}, \ldots, n_{k} \geq 0} \frac{\left(-q^{\ell+\frac{1}{2}}\right)_{N_{1}} q^{-\left(\ell+\frac{1}{2}\right) N_{1}+(2 \ell+1)\left(N_{1}+N_{2}+\cdots+N_{k}\right)+\frac{N_{1}\left(N_{1}-1\right)}{2}+N_{2}^{2}+N_{3}^{2}+\cdots+N_{k}^{2}-N_{2}-N_{3}-\cdots-N_{i}}}{(q)_{n_{1}}(q)_{n_{2}} \cdots(q)_{n_{k}}\left(q^{2 \ell+1}\right)_{n_{k}}} \\
= & \frac{\left(-q^{\ell+\frac{1}{2}}\right)_{\infty}}{\left(q^{2 \ell+2}\right)_{\infty}} \sum_{n=0}^{\infty} \frac{q^{-\left(\ell+\frac{1}{2}\right) n+(2 \ell+1) k n+\left(k-\frac{1}{2}\right) n^{2}+\left(\frac{1}{2}-i\right) n+n^{2}+2 \ell n}}{\left(1-q^{2 \ell+1}\right)} \\
& -\frac{\left(-q^{\ell+\frac{1}{2}}\right)_{\infty}}{\left(q^{2 \ell+2}\right)_{\infty}} \sum_{n=1}^{\infty} \frac{q^{-\left(\ell+\frac{1}{2}\right) n+(2 \ell+1)(k n+i-k+1)+\left(k-\frac{1}{2}\right) n^{2}+\left(\frac{5}{2}+i-2 k\right) n+k-i-2+(n-1)^{2}+2 \ell(n-1)}}{\left(1-q^{2 \ell+1}\right)} \\
= & \frac{\left(-q^{\ell+\frac{1}{2}}\right)_{\infty}}{\left(q^{2 \ell+1}\right)_{\infty}} \sum_{n=0}^{\infty} q^{\left(k+\frac{1}{2}\right) n^{2}+(2 k \ell+k-i+\ell) n}-\frac{\left(-q^{\ell+\frac{1}{2}}\right)_{\infty}}{\left(q^{2 \ell+1}\right)_{\infty}} \sum_{n=1}^{\infty} q^{\left(k+\frac{1}{2}\right) n^{2}+(2 k \ell-k+i+\ell) n+2 \ell(i-k)}
\end{aligned}
$$




$$
=\frac{\left(-q^{\ell+\frac{1}{2}}\right)_{\infty}}{\left(q^{2 \ell+1}\right)_{\infty}}\left(\sum_{n \geq \ell}-\sum_{n \leq-\ell-1}\right) q^{\left(k+\frac{1}{2}\right)\left(n+\frac{k-i}{2 k+1}\right)^{2}-\frac{(2 k \ell+k-i+\ell)^{2}}{4 k+2}} .
$$

The claimed identity then follows by multiplying the far extremes of the above identity by $\frac{\left(-q^{\frac{1}{2}}\right)_{\ell}}{(q)_{2 \ell}}$.

Remark. Proposition 3.1 gives $q$-hypergeometric expressions for characters for irreducible modules of the $N=1$ super-singlet vertex algebra (see (1.3) ).

Next we have a similar identity related to the $\epsilon=\frac{1}{2}$ case of the series discussed in the introduction.

Proposition 3.2. Suppose $i, k, \ell \in \mathbb{Z}, k \geq 1$, and $0 \leq i \leq k$. Then

$$
\begin{aligned}
& \frac{(-q)_{\infty}}{(q)_{\infty}}\left(\sum_{n \geq|\ell|}-\sum_{n \leq-|\ell|-1}\right) q^{\left(k+\frac{1}{2}\right)\left(n+\frac{2 i-1}{4 k+2}\right)^{2}-\frac{\left(2 k \ell+i+\ell-\frac{1}{2}\right)^{2}}{4 k+2}}\left(1+q^{n}\right) \\
& =\sum_{n_{1}, n_{2}, \ldots, n_{k} \geq 0} \frac{q^{\frac{N_{1}\left(N_{1}+1\right)}{2}+N_{2}^{2}+N_{3}^{2}+\cdots+N_{k}^{2}+\ell N_{1}+(2 \ell+1)\left(N_{2}+N_{3}+\cdots+N_{k}\right)-N_{1}-N_{2}-\cdots-N_{k-i}(-q)_{N_{1}+\ell}}}{(q)_{n_{1}}(q)_{n_{2}} \cdots(q)_{n_{k}}(q)_{n_{k}+2 \ell}} .
\end{aligned}
$$

Furthermore, the $i=k$ case simplifies as

$$
\begin{aligned}
& \frac{(-q)_{\infty}}{(q)_{\infty}}\left(\sum_{n \geq|\ell|}-\sum_{n \leq-|\ell|-1}\right) q^{\left(k+\frac{1}{2}\right)\left(n+\frac{2 k-1}{4 k+2}\right)^{2}-\frac{\left(2 k \ell+k+\ell-\frac{1}{2}\right)^{2}}{4 k+2}} \\
& =\sum_{n_{1}, n_{2}, \ldots, n_{k} \geq 0} \frac{q^{\frac{N_{1}\left(N_{1}+1\right)}{2}+N_{2}^{2}+N_{3}^{2}+\cdots+N_{k}^{2}+\ell N_{1}+(2 \ell+1)\left(N_{2}+N_{3}+\cdots+N_{k}\right)}(-q)_{N_{1}+\ell}}{(q)_{n_{1}}(q)_{n_{2}} \cdots(q)_{n_{k}}(q)_{n_{k}+2 \ell}}
\end{aligned}
$$

Proof: As with the proof of Proposition [3.1, we first verify that the identity for negative $\ell$ follows from the identity for positive $\ell$. Assuming that the identity holds for positive values of $\ell$, we then have for negative values of $\ell$ that

$$
\begin{aligned}
& \sum_{n_{1}, n_{2}, \ldots, n_{k} \geq 0} \frac{q^{\frac{N_{1}\left(N_{1}+1\right)}{2}+N_{2}^{2}+N_{3}^{2}+\cdots+N_{k}^{2}+\ell N_{1}+(2 \ell+1)\left(N_{2}+N_{3}+\cdots+N_{k}\right)-N_{1}-N_{2}-\cdots-N_{k-i}(-q)_{N_{1}+\ell}}}{(q)_{n_{1}}(q)_{n_{2}} \cdots(q)_{n_{k}}(q)_{n_{k}+2 \ell}} \\
= & \sum_{n_{1}, n_{2}, \ldots, n_{k} \geq 0} \frac{q^{\frac{\left(N_{1}-2 \ell\right)\left(N_{1}+1-2 \ell\right)}{2}+\left(N_{2}-2 \ell\right)^{2}+\left(N_{3}-2 \ell\right)^{2}+\cdots+\left(N_{k}-2 \ell\right)^{2}}(-q)_{N_{1}-\ell}}{(q)_{n_{1}}(q)_{n_{2}} \cdots(q)_{n_{k}}(q)_{n_{k}-2 \ell}} \\
& \times \sum_{n_{1}, n_{2}, \ldots, n_{k} \geq 0} \frac{q^{\ell\left(N_{1}-2 \ell\right)+(2 \ell+1)\left(N_{2}+N_{3}+\cdots+N_{k}-(k-1) 2 \ell\right)-N_{1}-N_{2}-\cdots-N_{k-i}+(k-i) 2 \ell}}{(q)_{n_{1}}(q)_{n_{2}} \cdots(q)_{n_{k}}(q)_{n_{k}-2 \ell}} \\
= & \frac{(-q)_{\infty}}{(q)_{\infty}}\left(\sum_{n \geq-\ell}-\sum_{n \leq \ell-1}\right) q^{\left(k+\frac{1}{2}\right)\left(n+\frac{2 i-1}{4 k+2}\right)^{2}-\frac{\left(-2 k \ell+i-\ell-\frac{1}{2}\right)^{2}}{4 k+2}+\ell-2 i \ell}\left(1+q^{n}\right) \\
= & \frac{(-q)_{\infty}}{(q)_{\infty}}\left(\sum_{n \geq|\ell|}-\sum_{n \leq-|\ell|-1}\right) q^{\left(k+\frac{1}{2}\right)\left(n+\frac{2 i-1}{4 k+2}\right)^{2}-\frac{\left(2 k \ell+i+\ell-\frac{1}{2}\right)^{2}}{4 k+2}}\left(1+q^{n}\right) .
\end{aligned}
$$


As such, we need only consider the case where $\ell \geq 0$.

We prove this identity after the change of variable $i \mapsto k-i$. We apply (2.2) with $\rho \mapsto-q^{\ell+1}$ to the Bailey pair in (2.4) with $a=q^{2 \ell+1}$. Doing so yields

$$
\begin{aligned}
& \sum_{n_{1}, n_{2}, \ldots, n_{k} \geq 0} \frac{\left(-q^{\ell+1}\right)_{N_{1}} q^{-(\ell+1) N_{1}+(2 \ell+1)\left(N_{1}+N_{2}+\cdots+N_{k}\right)+\frac{N_{1}\left(N_{1}-1\right)}{2}+N_{2}^{2}+N_{3}^{2}+\cdots+N_{k}^{2}-N_{2}-N_{3}-\cdots-N_{i}}}{(q)_{n_{1}}(q)_{n_{2}} \cdots(q)_{n_{k}}\left(q^{2 \ell+1}\right)_{n_{k}}} \\
= & \sum_{n_{1}, n_{2}, \ldots, n_{k} \geq 0} \frac{q^{\frac{N_{1}\left(N_{1}+1\right)}{2}+N_{2}^{2}+N_{3}^{2}+\cdots+N_{k}^{2}+\ell N_{1}+(2 \ell+1)\left(N_{2}+N_{3}+\cdots+N_{k}\right)-N_{1}-N_{2}-\cdots-N_{i}}\left(-q^{\ell+1}\right)_{N_{1}}}{(q)_{n_{1}}(q)_{n_{2}} \cdots(q)_{n_{k}}\left(q^{2 \ell+1}\right)_{n_{k}}} \\
= & \frac{\left(-q^{\ell}\right)_{\infty}}{\left(q^{2 \ell+2}\right)_{\infty}} \sum_{n=0}^{\infty} \frac{\left(-q^{\ell+1}\right)_{n} q^{-(\ell+1) n+(2 \ell+1) k n+\left(k-\frac{1}{2}\right) n^{2}+\left(\frac{1}{2}-i\right) n+n^{2}+2 \ell n}}{\left(-q^{\ell}\right)_{n}\left(1-q^{2 \ell+1}\right)} \\
& -\frac{\left(-q^{\ell}\right)_{\infty}}{\left(q^{2 \ell+2}\right)_{\infty}} \sum_{n=1}^{\infty} \frac{\left(-q^{\ell+1}\right)_{n} q^{-(\ell+1) n+(2 \ell+1)(k n+i-k+1)+\left(k-\frac{1}{2}\right) n^{2}+\left(\frac{5}{2}+i-2 k\right) n+k-i-2+(n-1)^{2}+2 \ell(n-1)}}{\left(1-q^{2 \ell+1}\right)} \\
= & \frac{\left(-q^{\ell+1}\right)_{\infty}}{\left(q^{2 \ell+1}\right)_{\infty}} \sum_{n=0}^{\infty} q^{\left(k+\frac{1}{2}\right) n^{2}+\left(2 k \ell+k-i+\ell-\frac{1}{2}\right) n}\left(1+q^{n+\ell}\right) \\
& -\frac{\left(-q^{\ell+1}\right)_{\infty}}{\left(q^{2 \ell+1}\right)_{\infty}} \sum_{n=1}^{\infty} q^{\left(k+\frac{1}{2}\right) n^{2}+\left(2 k \ell-k+i+\ell-\frac{1}{2}\right) n+2 \ell(i-k)}\left(1+q^{n+\ell}\right) \\
= & \frac{\left(-q^{\ell+1}\right)_{\infty}}{\left(q^{2 \ell+1}\right)_{\infty}}\left(\sum_{n \geq \ell}-\sum_{n \leq-\ell-1}\right) q^{\left(k+\frac{1}{2}\right)\left(n+\frac{2 k-2 i-1}{4 k+2}\right)^{2}-\frac{\left(2 k \ell+k-i+\ell-\frac{1}{2}\right)^{2}}{4 k+2}}\left(1+q^{n}\right) .
\end{aligned}
$$

The claimed identity then follows. When $i=0$,

$$
\left(\sum_{n \geq \ell}-\sum_{n \leq-\ell-1}\right) q^{\left(k+\frac{1}{2}\right)\left(n+\frac{2 k-1}{4 k+2}\right)^{2}-\frac{\left(2 k \ell+k+\ell-\frac{1}{2}\right)^{2}}{4 k+2}+n}=\left(\sum_{n \geq 0}-\sum_{n \geq 0}\right) q^{\left(k+\frac{1}{2}\right) n^{2}+\left(2 k \ell+k+\ell+\frac{1}{2}\right) n+\ell}=0,
$$

which finishes the proof of the proposition.

Remark. It would be interesting to connect the series in Proposition 3.2 with characters of indecomposable modules for the $N=1$ singlet algebra.

\section{Modular Identities}

In this part we prove a family of $q$-series identities for certain theta-like series that can be expressed as linear combinations of unary theta functions of weight $\frac{1}{2}$ and $\frac{3}{2}$. Such objects appear in the study of logarithmic modular forms (e.g. modular forms that are sums of ordinary modular forms of different weight). This terminology originates in Logarithmic Conformal Field Theory, where characters of representations often exhibit this property [1, 2].

Lemma 4.1. Suppose $i, k \in \mathbb{Z}$ and $0 \leq i \leq k$. Then

$$
\sum_{n \in \mathbb{Z}}(2 n+1) q^{\frac{2 k+1}{2}\left(n+\frac{i}{2 k+1}\right)^{2}}=\sum_{n \in \mathbb{Z}} \operatorname{sgn}(n) q^{\frac{2 k+1}{2}\left(n+\frac{i}{2 k+1}\right)^{2}}+2 \sum_{\ell \geq 1}\left(\sum_{n \geq \ell}-\sum_{n \leq-\ell-1}\right) q^{\frac{2 k+1}{2}\left(n+\frac{i}{2 k+1}\right)^{2}} .
$$

Proof: We omit the proof as it is near trivial. 
Let $k \geq 2$ and let $A$ be the $(k+1) \times(k+1)$ matrix defined by

$$
A:=\left(\begin{array}{ccccccc}
\frac{3}{2} & -\frac{1}{2} & 0 & \ldots & \ldots & \ldots & \ldots \\
-\frac{1}{2} & 1 & -\frac{1}{2} & \ldots & \ldots & \ldots & \ldots \\
0 & -\frac{1}{2} & 1 & \ldots & \ldots & \ldots & \ldots \\
\ldots & \ldots & \ldots & \ldots & \ldots & \ldots & \ldots \\
\ldots & \ldots & \ldots & \ldots & 1 & -\frac{1}{2} & -\frac{1}{2} \\
\ldots & \ldots & \ldots & \ldots & -\frac{1}{2} & 1 & 0 \\
\ldots & \ldots & \ldots & \ldots & -\frac{1}{2} & 0 & 1
\end{array}\right) .
$$

We note that

$$
A=\frac{1}{2}\left(D_{k+1}+E_{1,1}\right),
$$

where $D_{k+1}$ is the Cartan matrix of $D$-type. It is easy to show

$$
A^{-1}=\left(\begin{array}{ccccccc}
1 & 1 & 1 & \ldots & 1 & \frac{1}{2} & \frac{1}{2} \\
1 & 3 & 3 & \ldots & 3 & \frac{3}{2} & \frac{3}{2} \\
1 & 3 & 5 & \ldots & 5 & \frac{5}{2} & \frac{5}{2} \\
\ldots & \ldots & \ldots & \ldots & \ldots & \ldots & \ldots \\
1 & 3 & 5 & \ldots & 2 k-3 & \frac{2 k-3}{2} & \frac{2 k-3}{2} \\
\frac{1}{2} & \frac{3}{2} & \frac{5}{2} & \ldots & \frac{2 k-3}{2} & \frac{2 k+1}{4} & \frac{2 k-3}{4} \\
\frac{1}{2} & \frac{3}{2} & \frac{5}{2} & \ldots & \frac{2 k-3}{2} & \frac{2 k-3}{4} & \frac{2 k+1}{4}
\end{array}\right)
$$

or in more compact form

$$
\left(A^{-1}\right)_{i, j}=\left\{\begin{array}{cc}
\min \{2 i-1,2 j-1\}, & 1 \leq i, j \leq k-1 \\
\frac{2 j-1}{2}, & 1 \leq j \leq k-1, k \leq i \leq k+1 \\
\frac{2 k+1}{4 k}, & i=j \in\{k, k+1\} \\
\frac{2 k-3}{4}, & i=k, j=k+1
\end{array}\right.
$$

For $k=1$, we let $A^{-1}=\left(\begin{array}{cc}\frac{3}{4} & -\frac{1}{4} \\ -\frac{1}{4} & \frac{3}{4}\end{array}\right)$.

Now we can state Göllnitz-Gordon-Andrews-type identities for the series in Lemma 4.1.

Theorem 4.2. Suppose $i \in \mathbb{Z}, k \in \mathbb{N}$, and $0 \leq i \leq k$. Then

$$
\begin{aligned}
& q^{-\frac{i^{2}}{2(2 k+1)}} \frac{\left(-q^{1 / 2}\right)_{\infty}}{(q)_{\infty}} \sum_{n \in \mathbb{Z}}(2 n+1) q^{\frac{2 k+1}{2}\left(n+\frac{i}{2 k+1}\right)^{2}} \\
= & \sum_{\substack{n_{1}, n_{2}, \ldots, n_{k+1} \geq 0 \\
n_{k} \equiv n_{k+1} \bmod 2}} \frac{q^{\frac{1}{2} \mathbf{n} \cdot A^{-1} \cdot \mathbf{n}^{T}+n_{k-i+1}+2 n_{k-i+2}+\cdots+(i-1) n_{k-1}+\frac{i}{2}\left(n_{k}+n_{k+1}\right)}\left(-q^{\frac{1}{2}}\right)_{n_{1}+n_{2}+\cdots+n_{k-1}+\frac{n_{k}+n_{k+1}}{2}}}{(q)_{n_{1}}(q)_{n_{2}} \cdots(q)_{n_{k+1}}}
\end{aligned}
$$

where $\mathbf{n}=\left(n_{1}, \ldots, n_{k+1}\right)$.

Proof: We first rewrite the identity in Proposition 3.1 as

$$
F_{\ell}(q):=q^{-\frac{i^{2}}{4 k+2}} \frac{\left(-q^{\frac{1}{2}}\right)_{\infty}}{(q)_{\infty}}\left(\sum_{n \geq|\ell|}-\sum_{n \leq-|\ell|-1}\right) q^{\left(k+\frac{1}{2}\right)\left(n+\frac{i}{2 k+1}\right)^{2}}
$$




$$
\begin{aligned}
& =\sum_{n_{1}, n_{2}, \ldots, n_{k} \geq 0} \frac{q^{\left(k+\frac{1}{2}\right) \ell^{2}+i \ell+\frac{N_{1}\left(N_{1}+1\right)}{2}+N_{2}^{2}+N_{3}^{2}+\cdots+N_{k}^{2}+\left(\ell+\frac{1}{2}\right) N_{1}+(2 \ell+1)\left(N_{2}+N_{3}+\cdots+N_{k}\right)-N_{1}-N_{2}-\cdots-N_{k-i}}}{(q)_{n_{1}}(q)_{n_{2}} \cdots(q)_{n_{k}}(q)_{n_{k}+2 \ell}} \\
& \quad \times\left(-q^{\frac{1}{2}}\right)_{N_{1}+\ell .}
\end{aligned}
$$

From formula (4.1) for $A^{-1}$, we easily get

$$
\begin{aligned}
\frac{1}{2}\left(n_{1}, \ldots, n_{k-1}, 0,0\right) \cdot A^{-1} \cdot\left(n_{1}, \ldots, n_{k-1}, 0,0\right)^{T} & =\frac{1}{2}\left(\sum_{i=1}^{k-1} n_{i}\right)^{2}+\sum_{j=2}^{k-1}\left(\sum_{i=j}^{k-1} n_{i}\right)^{2}, \\
\left(0, \ldots, 0, n_{k}, n_{k}+2 \ell\right) \cdot A^{-1} \cdot\left(n_{1}, \ldots, n_{k-1}, 0,0\right)^{T} & =\frac{1}{2} \sum_{i=1}^{k-1}(2 i-1) n_{i}\left(2 n_{k}+2 \ell\right), \\
\frac{1}{2}\left(0, \ldots, 0, n_{k}, n_{k}+2 \ell\right) \cdot A^{-1} \cdot\left(0, \ldots, 0, n_{k}, n_{k}+2 \ell\right)^{T} & =\frac{2 k+1}{8}\left(n_{k}^{2}+\left(n_{k}+2 \ell\right)^{2}\right) \\
& +\frac{2 k-3}{4} n_{k}\left(n_{k}+2 \ell\right) .
\end{aligned}
$$

We then have that

$$
\begin{aligned}
& \frac{1}{2}\left(n_{1}, n_{2}, \ldots, n_{k}, n_{k}+2 \ell\right) A^{-1}\left(n_{1}, n_{2}, \ldots, n_{k}, n_{k}+2 \ell\right)^{T} \\
& =\frac{1}{2} N_{1}^{2}+N_{2}^{2}+N_{3}^{2}+\cdots+N_{k}^{2}+\ell N_{1}+2 \ell\left(N_{2}+N_{3}+\cdots+N_{k}\right)+\left(k+\frac{1}{2}\right) \ell^{2} .
\end{aligned}
$$

As such,

$$
\begin{aligned}
& \sum_{\substack{n_{1}, n_{2}, \ldots, n_{k+1} \geq 0 \\
n_{k} \equiv n_{k+1}(\bmod 2)}} \frac{q^{\frac{1}{2} \mathbf{n} \cdot A^{-1} \cdot \mathbf{n}^{T}+n_{k-i+1}+2 n_{k-i+2}+\cdots+(i-1) n_{k-1}+\frac{i}{2}\left(n_{k}+n_{k+1}\right)}\left(-q^{\frac{1}{2}}\right)_{n_{1}+n_{2}+\cdots+n_{k-1}+\frac{n_{k}+n_{k+1}}{2}}}{(q)_{n_{1}}(q)_{n_{2}} \cdots(q)_{n_{k+1}}} \\
= & \sum_{\substack{n_{1}, n_{2}, \ldots, n_{k} \geq 0 \\
\ell \in \mathbb{Z}}} \frac{q^{\frac{1}{2} N_{1}^{2}+N_{2}^{2}+N_{3}^{2}+\cdots N_{k}^{2}+\ell N_{1}+2 \ell\left(N_{2}+N_{3}+\cdots+N_{k}\right)+\left(k+\frac{1}{2}\right) \ell^{2}+n_{k-i+1}+2 n_{k-i+2}+\cdots+(i-1) n_{k-1}+i n_{k}+i \ell}}{(q)_{n_{1}}(q)_{n_{2}} \cdots(q)_{n_{k}}(q)_{n_{k}+2 \ell}} \\
& \quad \sum_{n_{1}, n_{2}, \ldots, n_{k} \geq 0} \frac{q^{\left(k+\frac{1}{2}\right) \ell^{2}+i \ell+\frac{N_{1}\left(N_{1}+1\right)}{2}+N_{2}^{2}+N_{3}^{2}+\cdots+N_{k}^{2}+\left(\ell+\frac{1}{2}\right) N_{1}+(2 \ell+1)\left(N_{2}+N_{3}+\cdots+N_{k}\right)-N_{1}-N_{2}-\cdots-N_{k-i}}}{(q)_{n_{1}}(q)_{n_{2}} \cdots(q)_{n_{k}}(q)_{n_{k}+2 \ell}} \\
& \times\left(-q^{\left.\frac{1}{2}\right)_{N_{1}+\ell}}\right. \\
= & \sum_{\ell \in \mathbb{Z}} F_{\ell}(q)=q^{-\frac{i^{2}}{4 k+2}} \frac{\left(-q^{\frac{1}{2}}\right)_{\infty}}{(q)_{\infty}} \sum_{n \in \mathbb{Z}}(2 n+1) q^{\left(k+\frac{1}{2}\right)\left(n+\frac{i}{2 k+1}\right)^{2}},
\end{aligned}
$$

where the final equality is by Lemma 4.1. This proves the claim.

We record the simplest case with $i=k=1$.

Corollary 4.3. The following identity holds,

$$
q^{-1 / 6} \frac{\left(-q^{1 / 2}\right)_{\infty}}{(q)_{\infty}} \sum_{n \in \mathbb{Z}}(2 n+1) q^{\frac{3}{2}\left(n+\frac{1}{3}\right)^{2}}=\sum_{\substack{n_{1}, n_{2} \geq 0 \\ n_{1} \equiv n_{2} \underset{\bmod 2}{ }}} \frac{q^{\frac{3}{8} n_{1}^{2}+\frac{3}{8} n_{2}^{2}-\frac{1}{4} n_{1} n_{2}+\frac{1}{2}\left(n_{1}+n_{2}\right)}\left(-q^{1 / 2}\right)_{\frac{n_{1}+n_{2}}{2}}}{(q)_{n_{1}}(q)_{n_{2}}} .
$$


Remark. Notice that for $i=0$, the sum on the left-hand side side in Theorem 4.2 simplifies, so we can write

$$
\frac{\left(-q^{1 / 2}\right)_{\infty}}{(q)_{\infty}} \sum_{n \in \mathbb{Z}} q^{\left(k+\frac{1}{2}\right) n^{2}}=\sum_{\substack{n_{1}, n_{2}, \ldots, n_{k+1} \geq 0 \\ n_{k} \equiv n_{k+1} \bmod 2}} \frac{q^{\frac{1}{2} \boldsymbol{n}^{T} \cdot A^{-1} \cdot \boldsymbol{n}}\left(-q^{\frac{1}{2}}\right)_{n_{1}+n_{2}+\cdots+n_{k-1}+\frac{n_{k}+n_{k+1}}{2}}}{(q)_{n_{1}}(q)_{n_{2}} \cdots(q)_{n_{k+1}}} .
$$

It would be interesting to find a combinatorial description of this identity.

There are similar identities coming from Proposition 3.2 . The $i=k$ case is more elegant than the general case, and so we record it as a separate theorem below. In both cases, we omit the proofs as they are near identical to that of Theorem 4.2.

Theorem 4.4. For $k \in \mathbb{N}$,

$$
\begin{aligned}
& q^{-\frac{(2 k-1)^{2}}{8(2 k+1)}} \frac{(-q)_{\infty}}{(q)_{\infty}} \sum_{n \in \mathbb{Z}}(2 n+1) q^{\frac{2 k+1}{2}\left(n+\frac{2 k-1}{2(2 k+1)}\right)^{2}} \\
& =\sum_{\substack{n_{1}, n_{2}, \ldots, n_{k+1} \geq 0 \\
n_{k} \equiv n_{k+1} \bmod 2}} \frac{q^{\frac{1}{2} \boldsymbol{n}^{T} \cdot A^{-1} \cdot \boldsymbol{n}+\frac{1}{2} n_{1}+\frac{3}{2} n_{2}+\cdots+\frac{2 k-3}{2} n_{k-1}+\frac{2 k-1}{4}\left(n_{k}+n_{k+1}\right)}(-q)_{n_{1}+n_{2}+\cdots+n_{k-1}+\frac{n_{k}+n_{k+1}}{2}}}{(q)_{n_{1}}(q)_{n_{2}} \cdots(q)_{n_{k+1}}} .
\end{aligned}
$$

For general $0 \leq i \leq k$, we instead have the following.

Theorem 4.5. Suppose $i \in \mathbb{Z}, k \in \mathbb{N}$, and $0 \leq i \leq k$, then

$$
\begin{aligned}
& q^{-\frac{(2 i-1)^{2}}{8(2 k+1)}} \frac{(-q)_{\infty}}{(q)_{\infty}} \sum_{n \in \mathbb{Z}}(2 n+1) q^{\frac{2 k+1}{2}\left(n+\frac{2 i-1}{4 k+2}\right)^{2}}\left(1+q^{n}\right) \\
& =\sum_{\substack{n_{1}, n_{2}, \ldots, n_{k+1} \geq 0 \\
n_{k} \equiv n_{k+1} \text { mod } 2}} q^{\frac{1}{2} \boldsymbol{n} \cdot A^{-1} \cdot \boldsymbol{n}^{T}-\frac{1}{2}\left(n_{1}+n_{2}+\cdots+n_{k-i}\right)+\frac{1}{2} n_{k-i+1}+\frac{3}{2} n_{k-i+2}+\cdots+\frac{2 i-3}{2} n_{k-1}+\frac{2 i-1}{4}\left(n_{k}+n_{k+1}\right)} \\
& \times \frac{(-q)_{n_{1}+n_{2}+\cdots+n_{k-1}+\frac{n_{k}+n_{k+1}}{2}}}{(q)_{n_{1}}(q)_{n_{2}} \cdots(q)_{n_{k+1}}} .
\end{aligned}
$$

\section{FURTHER $q$-SERIES IDENTITIES}

As discussed in the introduction, identity (1.6), which can be obtained from the Fourier expansion of a meromorphic Jacobi form, gives an elegant expression for the Rogers false theta function. In this section, we first present a generalization of that identity.

Theorem 5.1. For $k \in \mathbb{N}$,

$$
\frac{\sum_{n \in \mathbb{Z}} \operatorname{sgn}(n) q^{(k+1) n^{2}+k n}}{(q)_{\infty}^{2 k}}=\sum_{n_{1}, n_{2}, \ldots, n_{2 k-1} \geq 0} \frac{q^{\sum_{i=1}^{2 k-2} n_{i} n_{i+1}+\sum_{i=1}^{2 k-1} n_{i}}}{(q)_{n_{1}}^{2}(q)_{n_{2}}^{2} \cdots(q)_{n_{2 k-1}}^{2}}
$$

We note that these series have an odd number of summation variables. Interestingly, with an even number of summation variables we get a family of modular identities.

Theorem 5.2. For $k \in \mathbb{N}$,

$$
\frac{\left(q, q^{2 k+2}, q^{2 k+3} ; q^{2 k+3}\right)}{(q)_{\infty}^{2 k+1}}=\sum_{n_{1}, n_{2}, \ldots, n_{2 k} \geq 0} \frac{q^{\sum_{i=1}^{2 k-1} n_{i} n_{i+1}+\sum_{i=1}^{2 k} n_{i}}}{(q)_{n_{1}}^{2}(q)_{n_{2}}^{2} \cdots(q)_{n_{2 k}}^{2}} .
$$


We should point out that Theorem 5.2 was first conjectured in [19] based on an analysis of Schur's indices in certain Argyres-Douglas theories in physics. We shall present a uniform proof of both theorems. We do so with a combination of $q$-series techniques and Bailey's lemma.

Lemma 5.3. For $k \in \mathbb{N}$,

$$
\begin{aligned}
& \sum_{n_{1}, n_{2}, \ldots, n_{k} \geq 0} \frac{q^{n_{1} n_{2}+n_{2} n_{3}+\cdots+n_{k-1} n_{k}+n_{1}+n_{2}+\cdots+n_{k}}}{(q)_{n_{1}}^{2}(q)_{n_{2}}^{2} \cdots(q)_{n_{k}}^{2}} \\
= & \frac{1}{(q)_{\infty}^{k+1}} \sum_{m_{1}, m_{2}, \ldots, m_{k} \geq 0} \frac{(-1)^{m_{1}+m_{2}+\cdots+m_{k}} q^{\frac{m_{1}\left(m_{1}+1\right)}{2}}+\frac{m_{2}\left(m_{2}+1\right)}{2}+\cdots+\frac{m_{k}\left(m_{k}+1\right)}{2}}{(q)_{m_{1}-m_{2}}(q)_{m_{2}-m_{3}} \cdots(q)_{m_{k-1}-m_{k}}}
\end{aligned}
$$

Proof: We prove the stronger result that

$$
\begin{aligned}
& \sum_{n_{1}, n_{2}, \ldots, n_{k} \geq 0} \frac{x_{2}^{n_{1}} x_{3}^{n_{2}} \cdots x_{k}^{n_{k-1}} q^{n_{1} n_{2}+n_{2} n_{3}+\cdots+n_{k-1} n_{k}+n_{1}+n_{2}+\cdots+n_{k}}}{\left(q, x_{1} q\right)_{n_{1}}\left(q, x_{2} q\right)_{n_{2}} \cdots\left(q, x_{k} q\right)_{n_{k}}} \\
= & \frac{1}{(q)_{\infty}} \sum_{m_{1}, m_{2}, \ldots, m_{k} \geq 0} \frac{(-1)^{m_{1}+m_{2}+\cdots+m_{k}} x_{1}^{m_{1}} x_{2}^{m_{2}} \cdots x_{k}^{m_{k}} q^{\frac{m_{1}\left(m_{1}+1\right)}{2}}+\frac{m_{2}\left(m_{2}+1\right)}{2}+\cdots+\frac{m_{k}\left(m_{k}+1\right)}{2}}{(q)_{m_{1}-m_{2}}(q)_{m_{2}-m_{3}} \cdots(q)_{m_{k-1}-m_{k}}\left(x_{1} q, x_{2} q, \ldots, x_{k} q\right)_{\infty}} .
\end{aligned}
$$

The lemma then follows from by setting $x_{1}=x_{2}=\cdots=x_{k}=1$. Multiplying the left-hand side of (5.1) by $\left(x_{1} q, x_{2} q, \ldots, x_{k} q\right)_{\infty}$ gives

$$
\begin{aligned}
& \sum_{n_{1}, n_{2}, \ldots, n_{k} \geq 0} \frac{x_{2}^{n_{1}} x_{3}^{n_{2}} \cdots x_{k}^{n_{k-1}} q^{n_{1} n_{2}+n_{2} n_{3}+\cdots+n_{k-1} n_{k}+n_{1}+n_{2}+\cdots+n_{k}}\left(x_{1} q^{n_{1}+1}, x_{2} q^{n_{2}+1}, \ldots, x_{k} q^{n_{k}+1}\right)_{\infty}}{(q)_{n_{1}}(q)_{n_{2}} \cdots(q)_{n_{k}}} \\
&= \sum_{\substack{n_{1}, n_{2}, \ldots, n_{k} \geq 0 \\
m_{1}, m_{2}, \ldots, m_{k} \geq 0}} \frac{(-1)^{m_{1}+m_{2}+\cdots+m_{k}} x_{1}^{m_{1}} x_{2}^{m_{2}+n_{1}} x_{3}^{m_{3}+n_{2}} \cdots x_{k}^{m_{k}+n_{k-1}} q^{n_{1} n_{2}+n_{2} n_{3}+\cdots+n_{k-1} n_{k}}}{(q)_{n_{1}}(q)_{n_{2}} \cdots(q)_{n_{k}}(q)_{m_{1}}(q)_{m_{2}} \cdots(q)_{m_{k}}} \\
&=\sum_{\substack{n_{1}, n_{2}, \ldots, n_{k} \geq 0 \\
m_{1}, m_{2}, \ldots, m_{k} \geq 0}} \frac{(-1)^{m_{1}+m_{2}+\cdots+m_{k}+n_{1}+n_{2}+\cdots+n_{k-1}} x_{1}^{m_{1}} x_{2}^{m_{2}} \cdots x_{k}^{m_{k}} q^{\frac{m_{1}\left(m_{1}+1\right)}{2}+\frac{m_{2}\left(m_{2}+1\right)}{2}+\cdots+\frac{m_{k}\left(m_{k}+1\right)}{2}}}{(q)_{n_{1}}(q)_{n_{2}} \cdots(q)_{n_{k}}(q)_{m_{1}}(q)_{m_{2}-n_{1}}(q)_{m_{3}-n_{2}} \cdots(q)_{m_{k}-n_{k-1}}} \\
& \times q^{\frac{m_{1}\left(m_{1}+1\right)}{2}+\frac{m_{2}\left(m_{2}+1\right)}{2}+\cdots+\frac{m_{k}\left(m_{k}+1\right)}{2}+\frac{n_{2}\left(n_{2}+1\right)}{2}+\cdots+\frac{n_{k-1}\left(n_{k-1}+1\right)}{2}+n_{k}+m_{1} n_{1}+m_{2}\left(n_{2}-n_{1}\right)+m_{3}\left(n_{3}-n_{2}\right)+\cdots+m_{k}\left(n_{k}-m_{k-1}\right),}
\end{aligned}
$$

where in the first equality we have expanded each $\left(x_{j} q\right)_{\infty}$ according to an identity of Euler,

$$
(z ; q)_{\infty}=\sum_{m \geq 0} \frac{(-1)^{m} z^{m} q^{\frac{m(m-1)}{2}}}{(q)_{m}},
$$

and in the second equality we have shifted the indices $m_{j} \mapsto m_{j}-n_{j-1}$ for $j \geq 2$ (since $(q)_{n}^{-1}=0$ when $n$ is negative, the index bounds are still valid).

We recall a certain finite analogue of the $q$-binomial theorem [8, Theorem 3.3] states that

$$
\sum_{n=0}^{m} \frac{(-1)^{n} z^{n} q^{\frac{n(n-1)}{2}}(q)_{m}}{(q)_{n}(q)_{m-n}}=(z)_{m} .
$$


As such the inner sums on $n_{j}$ in the final equality of (15.2), for $1 \leq j<k$, are

$$
\sum_{n_{j} \geq 0} \frac{(-1)^{n_{j}} q^{n_{j}\left(m_{j}-m_{j+1}+1\right)+\frac{n_{j}\left(n_{j}-1\right)}{2}}}{(q)_{n_{j}}(q)_{m_{j+1}-n_{j}}}=\frac{\left(q^{m_{j}-m_{j+1}+1}\right)_{m_{j+1}}}{(q)_{m_{j+1}}}=\frac{(q)_{m_{j}}}{(q)_{m_{j+1}}(q)_{m_{j}-m_{j+1}}} .
$$

The inner sum on $n_{k}$, by another identity of Euler, is instead

$$
\sum_{n_{k} \geq 0} \frac{q^{n_{k}\left(m_{k}+1\right)}}{(q)_{n_{k}}}=\frac{1}{\left(q^{m_{k}+1}\right)_{\infty}}
$$

Equation (5.1) then follows by plugging these back into (5.2) and performing some elementary rearrangements of the summands.

We are now in a position to state and prove the general identity that encapsulates both Theorem 5.1 and Theorem 5.2 .

Theorem 5.4. For $k \in \mathbb{N}$,

$$
\begin{aligned}
& \sum_{n_{1}, n_{2}, \ldots, n_{k} \geq 0} \frac{q^{n_{1} n_{2}+n_{2} n_{3}+\cdots+n_{k-1} n_{k}+n_{1}+n_{2}+\cdots+n_{k}}}{(q)_{n_{1}}^{2}(q)_{n_{2}}^{2} \cdots(q)_{n_{k}}^{2}} \\
= & \frac{1}{(q)_{\infty}^{k+1}}\left(\sum_{n \geq 0}+(-1)^{k} \sum_{n<0}\right)(-1)^{n(k+1)} q^{\frac{(k+3) n^{2}+(k+1) n}{2}} .
\end{aligned}
$$

Proof: By applying the version of Bailey's lemma stated in (2.1) to the Bailey pair defined in (2.3), we find that Lemma 5.3 implies

$$
\begin{aligned}
& \sum_{n_{1}, n_{2}, \ldots, n_{k} \geq 0} \frac{q^{n_{1} n_{2}+n_{2} n_{3}+\cdots+n_{k-1} n_{k}+n_{1}+n_{2}+\cdots+n_{k}}}{(q)_{n_{1}}^{2}(q)_{n_{2}}^{2} \cdots(q)_{n_{k}}^{2}}=\frac{1}{(q)_{\infty}^{k+1}} \sum_{n \geq 0}(-1)^{n(k+1)} q^{\frac{(k+3) n^{2}+(k+1) n}{2}}\left(1-q^{2 n+1}\right) \\
= & \frac{1}{(q)_{\infty}^{k+1}}\left(\sum_{n \geq 0}+(-1)^{k} \sum_{n<0}\right)(-1)^{n(k+1)} q^{\frac{(k+3) n^{2}+(k+1) n}{2}} .
\end{aligned}
$$

We find that Theorem 5.4 establishes Thereom 5.1 and 5.2. In particular, Theorem 5.1 immediately follows from the statement of the theorem by letting $k \mapsto 2 k-1$. For Theorem 5.2, we let $k \mapsto 2 k$ in Theorem 5.4 and sum the series by using the Jacobi triple product identity to find that

$$
\sum_{n \in \mathbb{Z}}(-1)^{n} q^{\frac{(2 k+3) n^{2}+(2 k+1) n}{2}}=\sum_{n \in \mathbb{Z}}(-1)^{n} q^{\frac{(2 k+3) n^{2}-(2 k+1) n}{2}}=\left(q, q^{2 k+2}, q^{2 k+3} ; q^{2 k+3}\right)_{\infty} .
$$

\section{Classical Dilogarithm identities}

It is known that modular $q$-hypergeometric identities give rise to identities for the Rogers dilogarithm function [34, 37, 38, 48]; see also [42]. This is the case with characters of modules of rational vertex algebras (or conformal field theories) as they can be often expressed as $n$-fold $q$-hypergeometric series. 
Let $A=\left(A_{i, j}\right)$ be a symmetric positive definite $k \times k$ matrix with rational entries, $B \in \mathbb{Q}^{k}$, $C \in \mathbb{Q}$, and

$$
F_{A, B, C}(q):=\sum_{\boldsymbol{n}=\left(n_{1}, n_{2}, \ldots, n_{k}\right) \geq 0} \frac{q^{\frac{1}{2} \boldsymbol{n}^{T} A \boldsymbol{n}+B \boldsymbol{n}+C}}{(q)_{n_{1}}(q)_{n_{2}} \cdots(q)_{n_{k}}} .
$$

This is an example of a multi $q$-hypergeometric series sometimes called a Nahm sum. According to [32, 34, 37, 38], using the saddle point method, one can show that its asymptotic expansion (as $t \rightarrow 0^{+}, q=e^{-t}$ ) is given by:

$$
\begin{gathered}
F_{A, B, C}\left(e^{-t}\right) e^{-\alpha / t} \sim \beta e^{-\gamma t}(1+O(t)), \\
\alpha:=\sum_{i=1}^{k}\left(L(1)-L\left(Q_{i}\right)\right),
\end{gathered}
$$

where

$$
\mathrm{L}(x):=\mathrm{Li}_{2}(x)+\frac{1}{2} \log (x) \log (1-x), x \in(0,1),
$$

denotes Rogers' dilogarithm function and

$$
\operatorname{Li}_{2}(x)=\sum_{n \geq 1} \frac{x^{n}}{n^{2}}
$$

is the classical dilogarithm 1 , and $Q_{i} \in(0,1)$ are unique solutions of the TBA (thermodynamic Bethe ansatz) equation

$$
\left(1-Q_{i}\right)=\prod_{j=1}^{k} Q_{j}^{A_{i, j}}
$$

A detailed treatment of this result can be found in M. Vlasenko and S. Zwegers's recent paper [42].

We would like to extend this idea to our false theta function identities.

Lemma 6.1. Let $A$ be a positive definite real symmetric $k \times k$ matrix. Then

$$
\left(1-Q_{k}\right)^{2}=\prod_{j=1}^{k} Q_{j}^{A_{k, j}}, \quad\left(1-Q_{i}\right)=\prod_{j=1}^{k} Q_{j}^{A_{i, j}}, 1 \leq i \leq k-1
$$

has a unique solution inside $(0,1)$.

Proof: The proof follows along the lines of [42, Lemma 2.1]. The only modification required is to choose

$$
f_{A}(\mathbf{x})=\frac{1}{2} \mathbf{x}^{T} A \mathbf{x}+2 \operatorname{Li}_{2}\left(\operatorname{Exp}\left(-x_{k}\right)\right)+\sum_{i=1}^{k-1} \operatorname{Li}_{2}\left(\operatorname{Exp}\left(-x_{i}\right)\right),
$$

and proceed as in [42].

${ }^{1} L(x)$ can be extended to $x=0$ and $x=1 ; L(0)=0, L(1)=\frac{\pi^{2}}{6}$ 
6.1. Even case. Recall (for $k \geq 1$ )

$$
F(q):=\frac{q^{-\frac{k^{2}}{4(k+1)}}}{(q ; q)_{\infty}} \sum_{n \in \mathbb{Z}} \operatorname{sgn}(n) q^{(k+1)\left(n+\frac{k}{2(k+1)}\right)^{2}}=\sum_{n_{1}, n_{2}, \ldots, n_{k} \geq 0} \frac{q^{N_{1}^{2}+N_{2}^{2}+\cdots+N_{k}^{2}+N_{1}+N_{2}+\cdots+N_{k}}}{(q)_{n_{k}}^{2}(q)_{n_{1}}(q)_{n_{2}} \cdots(q)_{n_{k-1}}} .
$$

We let $f(q)=\sum_{n \in \mathbb{Z}} \operatorname{sgn}(n) q^{k\left(n+\frac{k-1}{2 k}\right)^{2}}$. It is known that (as $t \rightarrow 0^{+}$) (e.g. [47, Proposition $6.5])$

$$
f\left(e^{-t}\right) \sim \frac{1}{k}+O(t)
$$

Therefore

$$
e^{-\frac{\pi^{2}}{6 t}} F\left(e^{-t}\right) \sim \sqrt{\frac{t}{2 \pi}}\left(\frac{1}{k}+O(t)\right)
$$

where we have used the well-known behavior $\eta\left(e^{-t}\right)=\left.q^{1 / 24}(q)_{\infty}\right|_{q=e^{-t}} \sim \sqrt{\frac{2 \pi}{t}} e^{-\frac{\pi^{2}}{6 t}}$. Now we look at the $q$-hypergeometric side. Because of the $\frac{1}{(q)_{n_{k}}^{2}}$ we are led to a system of $k \times k$ equations (cf. [42])

$$
\begin{aligned}
\left(1-Q_{i}\right) & =\prod_{j \geq 1} Q_{j}^{B_{i, j}}, \quad 1 \leq i \leq k-1 \\
\left(1-Q_{k}\right)^{2} & =\prod_{j \geq 1} Q_{j}^{B_{k, j}}
\end{aligned}
$$

where $B=\left(B_{i, j}\right)=(2 \min (i, j))$ is the matrix corresponding to the quadratic form in the exponent of the RHS in (6.3).

Applying the procedure in [42] for computing the asymptotic exponent $\alpha$ of $F(q)$ in terms of $L(x)$, with slight modifications due to the squared factor $(q)_{n_{k}}^{2}$, gives the following identity.

Proposition 6.2. For $k \geq 2$, let $\left\{Q_{i}\right\}$ denote the unique solution of (6.4) inside the interval $(0,1)$. Then

$$
2\left(L(1)-L\left(Q_{k}\right)\right)+\sum_{r \geq 1}^{k-1}\left(L(1)-L\left(Q_{r}\right)\right)=\frac{\pi^{2}}{6} .
$$

Proof: It is not hard to see that $Q_{k}=\frac{k}{k+1}, Q_{1}=1-\frac{1}{2^{2}}, \ldots, Q_{k-1}=1-\frac{1}{k^{2}}$ is the solution of (6.4) inside $(0,1)$. Using $L(x)+L(1-x)=L(1)$, identity (6.5) is equivalent to

$$
2 L\left(\frac{1}{k+1}\right)+\sum_{r \geq 2}^{k} L\left(\frac{1}{r^{2}}\right)=\frac{\pi^{2}}{6} .
$$

The last identity is known and mentioned in [34, Exercise 6]. It can be easily proven by induction using the relation

$$
L\left(1-\frac{1}{x^{2}}\right)+2\left(L\left(\frac{1}{x}\right)+L\left(\frac{x}{x+1}\right)\right)=\frac{\pi^{2}}{2}
$$

with $x=k$, a consequence of the Five-Term relation for Rogers' dilogarithm [34, Formula $(1.4)]$. 
6.2. $N=1$ super identities. In this part we connect the $q$-series identities

$$
\begin{aligned}
& q^{-\frac{k^{2}}{2(2 k+1)}} \frac{\left(-q^{1 / 2}\right)_{\infty}}{(q)_{\infty}} \sum_{n \in \mathbb{Z}} \operatorname{sgn}(n) q^{\frac{2 k+1}{2}\left(n+\frac{k}{2 k+1}\right)^{2}} \\
& =\sum_{n_{1}, n_{2}, \ldots, n_{k} \geq 0} \frac{q^{\frac{N_{1}^{2}}{2}+N_{2}^{2}+N_{3}^{2}+\cdots+N_{k}^{2}+N_{1}+N_{2}+\cdots+N_{k}}\left(-q^{1 / 2}\right)_{N_{1}}}{(q)_{n_{k}}^{2}(q)_{n_{1}}(q)_{n_{2}} \cdots(q)_{n_{k-1}}}
\end{aligned}
$$

with dilogarithm identities. Here the situation is slightly more complicated due to the additional $q$-Euler factor, $\left(-q^{1 / 2}\right)_{N_{1}}$, appearing in the numerator. Observe that the leading asymptotics of the left-hand side in (6.8) is easily determined. After letting $q=e^{-t}$, it grows as $C e^{\frac{\pi^{2}}{4 t}}$ as $t \rightarrow 0^{+}$, which follows from using $q^{-\frac{1}{48}}\left(-q^{\frac{1}{2}}\right)_{\infty}=\frac{\eta(\tau)^{2}}{\eta\left(\frac{\tau}{2}\right) \eta(2 \tau)}$. Therefore we expect a family of identities for Rogers' dilogarithm as in (6.6), where the right-hand side equals $\frac{\pi^{2}}{4}$.

Proposition 6.3. For $k \in \mathbb{N}$,

$$
2 L\left(\frac{2}{2 k+1}\right)+\sum_{r=1}^{k-1} L\left(\frac{4}{(2 r+1)^{2}}\right)+L\left(\frac{3}{4}\right)-L\left(\frac{1}{2}\right)=\frac{\pi^{2}}{4} .
$$

Proof: As in [32], we first write

$$
\begin{aligned}
F(q) & :=\sum_{n_{1}, n_{2}, \ldots, n_{k} \geq 0} \frac{q^{\frac{N_{1}^{2}}{2}+N_{2}^{2}+N_{3}^{2}+\cdots+N_{k}^{2}+N_{1}+N_{2}+\cdots+N_{k}}\left(-q^{1 / 2}\right)_{N_{1}}}{(q)_{n_{k}}^{2}(q)_{n_{1}}(q)_{n_{2}} \cdots(q)_{n_{k-1}}} \\
& =\sum_{n_{1}, n_{2}, \ldots, n_{k+1} \geq 0} \frac{q^{N_{1}^{2}+N_{2}^{2}+\cdots+N_{k}^{2}+N_{1}+N_{2}+\cdots+N_{k}-N_{1} n_{k+1}+\frac{n_{k+1}^{2}}{2}}}{(q)_{n_{k}}^{2}(q)_{n_{1}}(q)_{n_{2}} \cdots(q)_{n_{k-1}}}\left[\begin{array}{c}
N_{1} \\
n_{k+1}
\end{array}\right]_{q},
\end{aligned}
$$

where $\left[\begin{array}{c}m \\ n\end{array}\right]_{q}:=\frac{(q)_{m}}{(q)_{n}(q)_{m-n}}$ are the $q$-binomial coefficients. For both multi-sums above, $N_{j}=$ $n_{j}+n_{j+1}+\cdots+n_{k}$, in particular $N_{j}$ does not end with $n_{k+1}$ in the latter sum. As before, saddle point analysis gives the system of equations:

$$
\begin{aligned}
\left(1-Q_{k}\right)^{2} & =\prod_{j=1}^{k} Q_{j}^{B_{k, j}} Q_{k+1}^{-1}, \\
\left(1-Q_{i}\right) & =\prod_{j=1}^{k} Q_{j}^{B_{i, j}} Q_{k+1}^{-1}, \quad 1 \leq i \leq k-1, \\
\left(1-Q_{k+1}\right) & =Q_{1}^{-1} \cdots Q_{k}^{-1} Q_{k+1} .
\end{aligned}
$$

As argued in [32, Section 5], specifically their formula (5.10), we get

$$
2\left(L(1)-L\left(Q_{k}\right)\right)+\left(L(1)-L\left(Q_{k+1}\right)\right)+\sum_{i=1}^{k-1}\left(L(1)-L\left(Q_{i}\right)\right)-\left(L(1)-L\left(\frac{1}{2}\right)\right)=\frac{\pi^{2}}{4},
$$

with the extra term $L(1)-L\left(\frac{1}{2}\right)=L\left(\frac{1}{2}\right)=\frac{\pi^{2}}{12}$ coming from the factor $\left(-q^{1 / 2}\right)_{N_{1}}$ in the sum. It is easy to check that

$$
Q_{k}=\frac{2 k-1}{2 k+1}, Q_{r}=1-\frac{4}{(2 r+1)^{2}} \quad(1 \leq r \leq k-1), \quad Q_{k+1}=\frac{1}{4}
$$


is a solution of the above system. The proof then follows. Alternatively, we can prove (6.9) directly by induction on $k$ using relation (6.7).

Remark. We note that taking $k \rightarrow+\infty$ in (6.9) leads to

$$
\sum_{r \geq 1} L\left(\frac{4}{(2 r+1)^{2}}\right)+L\left(\frac{3}{4}\right)-L\left(\frac{1}{2}\right)=\frac{\pi^{2}}{4}
$$

This identity can be viewed as a limiting case of Melzer's dilogarithm identity coming from $N=1$ superconformal characters [36, Formula (3.12)]:

$$
\sum_{r=1}^{k-1} L\left(\frac{\sin ^{2} \frac{\pi}{2 k}}{\sin ^{2} \frac{(2 r+1) \pi}{4 k}}\right)+L\left(1-\frac{1}{4 \cos ^{2} \frac{\pi}{4 k}}\right)-L\left(\frac{1}{2}\right)=\pi^{2} \frac{(k-1)}{4 k} .
$$

Indeed, formally taking $k \rightarrow+\infty$ in (6.11), we get (6.10) by L'hopital's rule.

6.3. Further Dilogarithm Identities. We are not aware of any $q$-series identities connecting

$$
\sum_{n_{1}, n_{2}, \ldots, n_{k} \geq 0} \frac{q^{\frac{N_{1}^{2}}{2}+N_{2}^{2}+N_{3}^{2}+\cdots+N_{k}^{2}+N_{1}+N_{2}+\cdots+N_{k}}}{(q)_{n_{k}}^{2}(q)_{n_{1}}(q)_{n_{2}} \cdots(q)_{n_{k-1}}}
$$

with a false theta-type $q$-series. The proposition below gives a dilogarithm identity that suggests a relation with a false theta series is plausible.

Proposition 6.4. For $k \in \mathbb{N}$, let $\left\{Q_{i}\right\}$ be the unique solution of

$$
\left(1-Q_{k}\right)^{2}=\prod_{1 \leq j \leq k} Q_{j}^{C_{k, j}}, \quad\left(1-Q_{i}\right)=\prod_{1 \leq j \leq k} Q_{j}^{C_{i, j}} ; \quad 1 \leq i \leq k-1,
$$

inside the interval $(0,1)$, where $\left(C_{i, j}\right)=(2 \min (i, j)-1)$ is the matrix corresponding to the quadratic form in the exponent of the q-hypergeometric series (6.12). Then

$$
2\left(L(1)-L\left(Q_{k}\right)\right)+\sum_{i=1}^{k-1}\left(L(1)-L\left(Q_{i}\right)\right)=\frac{\pi^{2}}{5} .
$$

Proof: Let

It is not hard to see that

$$
a=\frac{1}{2}(\sqrt{5}-1)
$$

$$
Q_{1}=a, Q_{2}=1-\frac{1}{(a+2)^{2}}, \ldots, Q_{k-1},=1-\frac{1}{(a+k-1)^{2}}, Q_{k}=1-\frac{1}{a+k}
$$

is the unique solution of $(\underline{6.13})$ inside $(0,1)$; when $k=1$ we have $Q_{1}=1-\frac{1}{a+1}$. The rest follows by induction on $k$ using the five-term relation as in Proposition 6.2.

\section{Final COMMENTS ON FUTURE WORK}

In a sequel to this paper [31], we plan to study $q$-series identities involving expressions with even "higher poles" (cf. Section 5)

$$
\sum_{n_{1}, n_{2}, \ldots, n_{k} \geq 0} \frac{q^{\sum_{i=1}^{k} n_{i}+\sum_{i=1}^{k-1} n_{i} n_{i+1}}}{(q)_{n_{1}}^{r_{1}}(q)_{n_{2}}^{r_{2}} \cdots(q)_{n_{k}}^{r_{k}}}
$$


We will also extend Theorems 5.1 and Theorem 5.2 to half-characteristics, where the relevant $q$-hypergeometric series are

$$
\sum_{n_{1}, n_{2}, \ldots, n_{k} \geq 0} \frac{q^{\sum_{i=1}^{k} n_{i}^{2}-\sum_{i=1}^{k-1} n_{i} n_{i+1}}\left(-q^{\epsilon}\right)_{n_{1}}}{(q)_{n_{1}}^{2}(q)_{n}^{2} \cdots(q)_{n_{k}}^{2}} .
$$

Moreover, motivated by [26], we will study identities for "inverted" $q$-hypergeometric terms, after the formal inversion $q \rightarrow q^{-1}$.

\section{REFERENCES}

[1] D. Adamović and A. Milas. The $N=1$ triplet vertex operator superalgebras: twisted sector. SIGMA Symmetry Integrability Geom. Methods Appl., 4: (2008) pp. Paper 087, 24.

[2] D. Adamović and A. Milas. The $N=1$ triplet vertex operator superalgebras. Comm. Math. Phys., 288(1): (2009) pp. 225-270.

[3] D. Adamović, A. Milas, and S. Sidoli. The $N=1$ super singlet vertex operator superalgebra. to appear.

[4] A. K. Agarwal, G. E. Andrews, and D. M. Bressoud. The Bailey lattice. J. Indian Math. Soc. (N.S.), 51: (1987) pp. 57-73 (1988).

[5] G. E. Andrews. Partitions and Durfee dissection. Amer. J. Math., 101(3): (1979) pp. 735-742.

[6] G. E. Andrews. Hecke modular forms and the Kac-Peterson identities. Trans. Amer. Math. Soc., 283(2): (1984) pp. 451-458.

[7] G. E. Andrews. q-Series: their development and application in analysis, number theory, combinatorics, physics, and computer algebra, vol. 66 of CBMS Regional Conference Series in Mathematics. Published for the Conference Board of the Mathematical Sciences, Washington, DC; by the American Mathematical Society, Providence, RI (1986).

[8] G. Andrews, The theory of partitions, Cambridge University Press, Cambridge, 1998.

[9] W. N. Bailey. Identities of the Rogers-Ramanujan type. Proc. London Math. Soc. (2), 50: (1948) pp. $1-10$.

[10] P. Beirne and R. Osburn. q-Series and tails of colored Jones polynomials. Indag. Math. (N.S.), 28(1): (2017) pp. 247-260.

[11] B. C. Berndt. Ramanujan's notebooks. Part III. Springer-Verlag, New York (1991).

[12] K. Bringmann, A. Folsom, K. Ono, and L. Rolen. Harmonic Maass forms and mock modular forms: theory and applications, vol. 64 of American Mathematical Society Colloquium Publications. American Mathematical Society, Providence, RI (2017).

[13] K. Bringmann, K. Hikami, and J. Lovejoy. On the modularity of the unified WRT invariants of certain Seifert manifolds. Adv. in Appl. Math., 46(1-4): (2011) pp. 86-93.

[14] K. Bringmann, J. Kaszian, and A. Milas. Higher depth quantum modular forms, multiple Eichler integrals, and $\mathfrak{s l}_{3}$ false theta functions. Res. Math. Sci., 6(2): (2019) pp. Paper No. $20,41$.

[15] K. Bringmann, K. Mahlburg, and A. Milas. Quantum modular forms and plumbing graphs of 3manifolds. J. Combin. Theory Ser. A, 170: (2020) pp. 105145, 32.

[16] K. Bringmann and A. Milas. W-algebras, false theta functions and quantum modular forms, I. Int. Math. Res. Not. IMRN, (21): (2015) pp. 11351-11387.

[17] K. Bringmann and A. Milas. W-algebras, higher rank false theta functions, and quantum dimensions. Selecta Math. (N.S.), 23(2): (2017) pp. 1249-1278.

[18] M. C. N. Cheng, S. Chun, F. Ferrari, S. Gukov, and S. M. Harrison. 3d modularity. J. High Energy Phys., (10): (2019) pp. 010, front matter+94.

[19] C. Córdova and S.-H. Shao. Schur indices, BPS particles, and Argyres-Douglas theories. J. High Energy Phys., (1): (2016) pp. 040, front matter+37.

[20] T. Creutzig and A. Milas. Higher rank partial and false theta functions and representation theory. Adv. Math., 314: (2017) pp. 203-227.

[21] L. D. Faddeev and R. M. Kashaev. Quantum dilogarithm. Modern Phys. Lett. A, 9(5): (1994) pp. $427-434$. 
[22] B. Feigin, E. Feigin, and I. Tipunin. Fermionic formulas for characters of $(1, p)$ logarithmic model in $\Phi_{2,1}$ quasiparticle realisation. In Exploring new structures and natural constructions in mathematical physics, vol. 61 of Adv. Stud. Pure Math., pp. 161-184. Math. Soc. Japan, Tokyo (2011).

[23] A. Folsom, K. Ono, and R. C. Rhoades. Mock theta functions and quantum modular forms. Forum Math. Pi, 1: (2013) pp. e2, 27.

[24] S. Garoufalidis and T. T. Q. Lê. Nahm sums, stability and the colored Jones polynomial. Res. Math. Sci., 2: (2015) pp. Art. 1, 55.

[25] G. Gasper and M. Rahman. Basic hypergeometric series, vol. 96 of Encyclopedia of Mathematics and its Applications. Cambridge University Press, Cambridge, second ed. (2004). With a foreword by Richard Askey.

[26] S. Gukov, D. Pei, P. Putrov, and C. Vafa. BPS spectra and 3-manifold invariants. arXiv e-prints, arXiv:1701.06567.

[27] M. Hajij. The tail of a quantum spin network. Ramanujan J., 40(1): (2016) pp. 135-176.

[28] K. Hikami. Quantum invariant, modular form, and lattice points. Int. Math. Res. Not., (3): (2005) pp. $121-154$.

[29] K. Hikami. On the quantum invariants for the spherical Seifert manifolds. Comm. Math. Phys., 268(2): (2006) pp. 285-319.

[30] K. Hikami and J. Lovejoy. Torus knots and quantum modular forms. Res. Math. Sci., 2: (2015) pp. Art. 2,15 .

[31] C. Jennings-Shaffer and A. Milas. Further q-series identities and conjectures relating false theta functions and characters. preprint, to appear in Contemporary Mathematics.

[32] R. Kedem, T. R. Klassen, B. M. McCoy, and E. Melzer. Fermionic sum representations for conformal field theory characters. Phys. Lett. B, 307(1-2): (1993) pp. 68-76.

[33] A. Keilthy and R. Osburn. Rogers-Ramanujan type identities for alternating knots. J. Number Theory, 161: (2016) pp. 255-280.

[34] A. N. Kirillov. Dilogarithm identities. 118, pp. 61-142 (1995). Quantum field theory, integrable models and beyond (Kyoto, 1994).

[35] R. Lawrence and D. Zagier. Modular forms and quantum invariants of 3-manifolds. pp. 93-107 (1999). Sir Michael Atiyah: a great mathematician of the twentieth century.

[36] E. Melzer. Supersymmetric analogs of the Gordon-Andrews identities, and related tba systems. arXiv e-prints, hep-th/9412154.

[37] W. Nahm. Conformal field theory and torsion elements of the Bloch group. In Frontiers in number theory, physics, and geometry. II, pp. 67-132. Springer, Berlin (2007).

[38] W. Nahm, A. Recknagel, and M. Terhoeven. Dilogarithm identities in conformal field theory. Modern Phys. Lett. A, 8(19): (1993) pp. 1835-1847.

[39] S. Sidoli. The $N=1$ Singlet Vertex Superalgebra $\overline{S M(1)}$, PhD thesis, SUNY-Albany: (2018)

[40] L. J. Slater. A new proof of Rogers's transformations of infinite series. Proc. London Math. Soc. (2), 53: (1951) pp. 460-475.

[41] J. Tannery and J. Molk Éléments de la théorie des fonctions elliptiques. Tome III:Calcul intégral. Première partie. Tome IV: Calcul intégral. Deuxième partie, Chelsea Publishing Co.: (1972)

[42] M. Vlasenko and S. Zwegers. Nahm's conjecture: asymptotic computations and counterexamples. Commun. Number Theory Phys., 5(3): (2011) pp. 617-642.

[43] S. O. Warnaar. Partial theta functions. I. Beyond the lost notebook. Proc. London Math. Soc. (3), 87(2): (2003) pp. 363-395.

[44] S. O. Warnaar. Proof of the Flohr-Grabow-Koehn conjectures for characters of logarithmic conformal field theory. J. Phys. A, 40(40): (2007) pp. 12243-12254.

[45] S. O. Warnaar. Partial theta functions. Encyclopedia Mathematica: preprint.

[46] S. O. Warnaar. personal communications.

[47] D. Zagier, The Mellin transform and related analytic techniques. appendix to E. Zeidler, Quantum Field Theory I: Basics in Mathematics and Physics. A Bridge Between Mathematicians and Physicists, pp. 305-323. Springer-Verlag (2006).

[48] D. Zagier. The dilogarithm function. In Frontiers in number theory, physics, and geometry. II, pp. 3-65. Springer, Berlin (2007). 
Department of Mathematics, University of Denver, 2390 S. York St. Denver, CO 80208. E-mail address: christopher.jennings-shaffer@du.edu

Department of Mathematics and Statistics, SUNY-Albany, 1400 Washington Avenue AlBANY, NY 12222 .

E-mail address: amilas@albany.edu 